\title{
Preliminary Study of Calcium Homeostasis Modulator 1 Involvement in Trigeminal Neuralgia
}

\author{
C. WANG, X.F. CHEN ${ }^{1 *}$, X.L. MA ${ }^{2}$, M. ZHANG ${ }^{3}$
}

Department of Oral Medicine, ${ }^{1}$ Department of Orthodontics, ${ }^{2}$ Department of Oral Surgery, Hebei Eye Hospital, No. 399, Quanbei East Street, Xingtai, Hebei 054001, China. ${ }^{3}$ Department of Radiotherapy, The First Hospital of Xingtai, Qiaodong District, Hebei, China.

Wang et al.: Calcium Homeostasis Modulator 1 in Trigeminal Neuralgia

\begin{abstract}
This study was to observe the changes in the expression of calcium homeostasis modulator 1 in trigeminal nucleus of trigeminal neuralgia rats with the infraorbital nerve-chronic constrictive nerve injury and to explore the role of calcium homeostasis modulator 1 in trigeminal neuralgia. Thirty Sprague Dawley rats were randomly assigned to 5 groups, control group, sham group, infraorbital nerve-chronic constrictive nerve injury group, ruthenium red group and normal saline group, with 6 rats in each group. All experimental rats were tested for pain behaviour $1 \mathrm{~d}$ before surgery, 1, 3, 5, 7, 9, 11 and $14 \mathrm{~d}$ after surgery, including the mechanical pain threshold of Von Frey filament in the trigeminal innervated skin area and number of faces captured in video recording. The expression of calcium homeostasis modulator 1 in the trigeminal spinal nucleus was detected on d 15 after surgery in all experimental groups. The expression of calcium homeostasis modulator 1 in the trigeminal spinal nucleus of the infraorbital nerve-chronic constrictive nerve injury group was significantly higher than that of the control and the sham group on the 3rd and 15th d after modelling. Intraperitoneal injection of ruthenium red, a calcium homeostasis modulator 1 inhibitor, increased the mechanical pain threshold of infraorbital nerve-chronic constrictive nerve injury rats and significantly reduced the number of scratches, but did not change the expression of calcium homeostasis modulator 1 in the trigeminal spinal nucleus. In conclusion, the expression of calcium homeostasis modulator 1 protein in the trigeminal spinal nucleus is involved in the central sensitization of trigeminal neuralgia pain. Moreover, the hyperalgesia can be improved by using calcium homeostasis modulator 1 inhibitor without affecting the calcium homeostasis modulator 1 expression.
\end{abstract}

Key words: Trigeminal neuralgia, spinal trigeminal nucleus, calcium homeostasis modulator 1, ruthenium red

Trigeminal neuralgia $(\mathrm{TN})$ or maxillofacial intractable pain is a common clinical problem. TN is a form of neuropathic pain with a major pathological change in the loss of trigeminal root axons and demyelination. Clinically, it often characterized by sudden electric shock-like burst pain and tactile hyperalgesia and because of repeated pain, the pain is severe and difficult to cure, which seriously affects the quality of life of patients ${ }^{[1]}$. Calcium homeostasis modulator 1 (CHM 1) is a cell surface calcium channel expressed in brain neurons. CHM 1 controls intracellular calcium signaling, which reduces the resistance of ion influx in sensory neurons and enhances the ion influx of neurons ${ }^{[2]}$. Thus, the excitability of neuronal cells is dramatically enhanced ${ }^{[3]}$. Whether CHM 1 is involved in the occurrence of $\mathrm{TN}$ has not been reported in the literature. The aim of this study was to investigate the role of CHM 1 in TN in the trigeminal spinal nucleus of rats with infraorbital nerve-chronic constrictive nerve injury (ION-CCI).

Adult male Sprague Dawley (SD) rats (200-220 g) were purchased from the Experimental Animal Center of Central South University. The experimental procedures conformed to institutional guidelines for the care and use of experimental animals and were approved by the Animal Management and Use Committee of the Heibei Key Laboratory of Ophthalmology.

ION-CCI was used to establish a trigeminal pain model as described previously ${ }^{[4]}$. In brief, the infraorbital nerve was loosely ligated with absorbable chromium gut. The ligation standard was that the diameter of the nerve was slightly thinned after ligation under the operating microscope, but the conduction could not be completely blocked and the blood circulation of the epicardium remained unobstructed. Thirty SD rats were randomly

*Address for correspondence 
divided into the control group, sham group, ION-CCI group, normal saline (NS) group (ION-CCI+NS) and ruthenium red (RR) group (ION-CCI+RR) with 6 rats in each group. The rats in the control group were not treated, and the surgical procedure of rats in the sham group was the same as the ION-CCI group except that the infraorbital nerve was not ligated. On d 9 after surgery, rats in the RR group were intraperitoneally injected with RR, a CHM 1 inhibitor, at a dose of $0.5 \mathrm{mg} / \mathrm{kg}$ per day, while the rats in the NS group was injected with an equal volume of NS. Determination of mechanical pain threshold was determined as follows, all rats were acclimated to the environment for $2 \mathrm{w}$ before pain behavioral measurements were performed $1 \mathrm{~d}$ before surgery (ION-CCI or sham surgery) and 1, 3, $5,7,9,11,14 \mathrm{~d}$ after treatment. According to Vos et al. ${ }^{[5]}$, the Von Frey filament is placed near the center of the whisker pad so that the filament is slightly curved. Each rat was stimulated at least 3 times from the minimum stimulation intensity, and the shortest interval between 2 stimulations was $30 \mathrm{~s}$ and the response was considered positive if one or more of the following behaviors were noticed. Rapid withdrawal response in rats or escape or attack response or the asymmetrical facial trimming behavior is characterized by more than 3 consecutive strokes of the stimulated facial area. The minimum value of the filament strength that causes a positive reaction in the rat is the mechanical pain threshold of the surgical side whisker pad. Measurements were not performed in the order of grouping, and the order of placement of rats was disrupted and random measurements were made by those who did not understand the experimental group of rats to reduce experimental errors.

Video recording of the number of scratches was made according to Spradley et al. ${ }^{[6]}$, rats were placed in a colorless transparent glass lattice that did not interfere with rats' random movement. Rats were allowed to adapt to the environment of the lattice in advance and they were allowed to move freely for $1 \mathrm{~h}$ before video recording was initiated. The video observation lasted for $30 \mathrm{~min}$. The observers were not informed of the grouping before the experiment and the number of scratches of each group of rats at the corresponding time points ( $1 \mathrm{~d}$ before surgery and 1, 3, 5, 7, 9, 11 and $14 \mathrm{~d}$ after surgery) was recorded. Quiet environment was maintained during the recording so as not to scare the rats into affecting the experimental results.

On the d 15 after surgery, the trigeminal nucleus of the rat was removed aseptically under the direction of a brain stereotaxic instrument. The lysate containing a protease inhibitor was added, the tissue was homogenized and placed in $4^{\circ}$. After centrifugation at $12000 \mathrm{rpm}$ for $15 \mathrm{~min}$, the protein content of the supernatant was determined using bicinchoninic acid quantification. The protein was then denatured with the loading buffer and dried at $100^{\circ}$ for $5 \mathrm{~min}$. After sodium dodecyl sulfate-polyacrylamide gel electrophoresis and electro transformation, the protein was transferred to a polyvinylidene fluoride membrane and blocked for $2 \mathrm{~h}$ with $5 \%$ skim milk. Rabbit anti-CALHM 1 primary antibody (1:1000) diluted with $5 \%$ TBST was added and left overnight at $4^{\circ}$. After washing the membrane, the mouse secondary antibody (1:5000) was separately added, and the membrane was then incubated at $37^{\circ}$ for $1 \mathrm{~h}$, and then subjected to enhanced chemiluminescence coloration and exposure. Semi-quantitative analysis was performed using $\beta$-actin as an internal control. Image $\mathrm{J}$ was used for gray scale analysis.

All data were expressed as mean \pm standard deviation. Two-way analysis of variance (ANOVA) was used to compare the mechanical pain threshold and video behavioral results of each group of rats, and comparison between groups was performed at the same time point. The difference of gray value of each group was analyzed by one-way ANOVA. The difference was statistically significant $(\mathrm{p}<0.05)$. The data were analyzed by GraphPad Prism 6 software.

From the $\mathrm{d} 1$ to $\mathrm{d} 3$ after surgery, rats in the ION-CCI group showed a brief and sharp increase in the threshold of mechanical pain, which was significantly higher than that of rats in the control group and sham group (fig. 1A). However, on d 5, the pain threshold in the ION-CCI group decreased slowly to the same level as the control group and the sham group. Pain sensitivity continued from d 9 and mechanical pain threshold reached a minimum at d 14 after surgery. The mechanical pain threshold of rats in the control and the sham group did not fluctuate much before and after operation, and there was no statistical difference between the two groups.

In the ION-CCI and the sham group, the frequency of scratching face increased significantly on the $\mathrm{d} 1$ and d 3 after surgery, which were both significantly higher than that in the control group. However, the number of face scratching in the sham group gradually decreased to the same level as that in the control group from $\mathrm{d} 3$. But in the ION-CCI group, the number of face scratching decreased slowly from d 3 to $\mathrm{d} 14$, which was significantly higher than that in the control and the 
sham group (fig. $1 \mathrm{~B}, \mathrm{p}<0.05$ ). These data showed that the TN model was constructed successfully.

To test whether CHM 1 was related to the occurrence of facial pain, the expression of CHM 1 in the lateral trigeminal nucleus of the rats in each group was estimated. As shown in fig. 2, compared with the sham group, CHM 1 began to increase significantly on $\mathrm{d} 3$ in ION-CCI rats undergoing infraorbital nerve ligation, and reached a peak on $\mathrm{d} 15$, which suggested that CHM 1 might play an important role in TN.

Additionally, RR was intraperitoneally injected to rats subjected to ION-CCI surgery from d 9 . As shown in fig. 3, after injection of RR, the threshold of mechanical pain in the RR group increased, while the number of scratches decreased, which indicated that RR could reduce the hyperalgesia of TN rats. However, on d 15, there was no significant difference in the expression of CHM 1 among the RR group, the ION-CCI group and the NS group, as shown in fig. 4. These results demonstrated that injection of $\mathrm{RR}$ can improve hyperalgesia in $\mathrm{TN}$ rats without affecting the CHM 1 expression.

There are many hypothesis on the pathogenesis of $\mathrm{TN}$, among which the following are more accepted by many researchers who studied $\mathrm{TN}^{[7]}$ : Peripheral causes are important pathological basis of $\mathrm{TN}$, while the central factors are also indispensable. At present, the most widely used model in the study of TN is ION-CCI. The
A

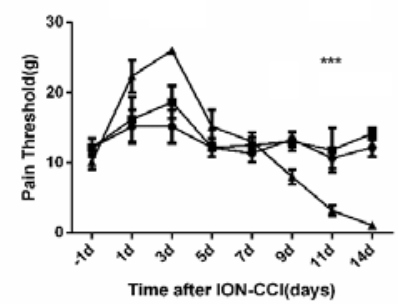

B

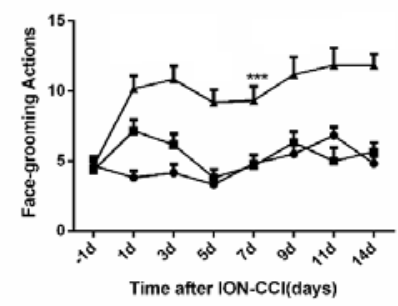

Fig. 1: Influence of ION-CCI pain threshold in rats Chronic constriction injury of the infraorbital nerve (ION-CCI) in rats produced hyperalgesia $A$. The threshold of mechanical pain in rats; $\mathrm{B}$. number of face scratches in rats. $* * * \mathbf{p}<0.001$, -๑- control, - - sham, - $\triangle-$ ION-CCI
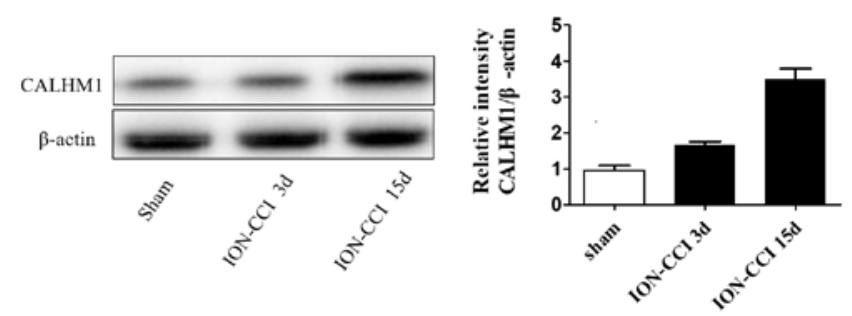

Fig. 2: CHM 1 expression in rats with ION-CCI

\section{A}
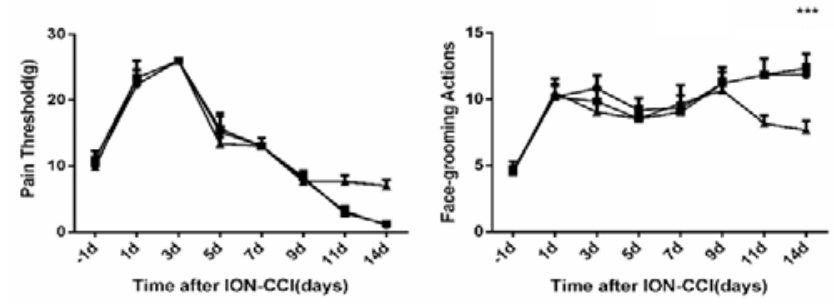

Fig. 3: Effect of RR injection on hyperalgesia in TN rats Injection of ruthenium red (RR) improved hyperalgesia in trigeminal neuralgia (TN) rats. A. threshold of mechanical pain in rats; $B$. number of face scratches in rats. $* * * p<0.001,-\bullet-$ ION-CCI, - $-\mathrm{NS},-\Delta-\mathrm{RuR}$
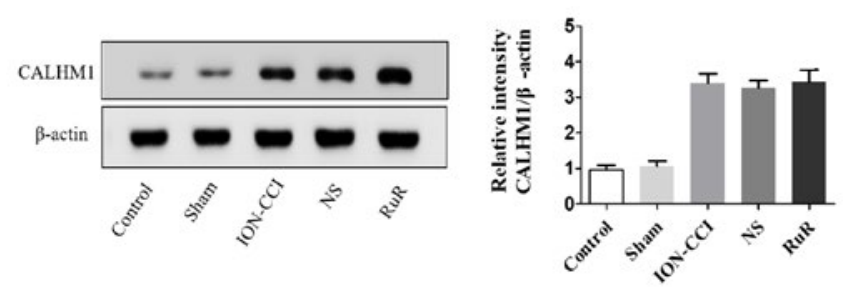

Fig. 4: Effect of RR on CHM 1 expression

$R R$ is ruthenium red and CHM 1 is the calcium homeostasis modulator 1

pain behavioral results in this study showed that rats with ION-CCI surgery exhibited painful dullness from the $\mathrm{d} 1$ to $\mathrm{d} 3$ after surgery. By d 5, it slowly dropped to the normal level. This result was consistent with the findings of Kajander and Bennet ${ }^{[8]}$, considering that the conduction of trigeminal nerve impulse in rats was affected after ligation. In this study, TN rats continued to show pain sensitivity on $\mathrm{d} 9$, and the mechanical pain threshold reached the lowest level on d 14 after surgery. This result was consistent with the findings reported by Vos et al. ${ }^{[5]}$.

In recent years, AD-related studies have shown that CHM 1 can affect neuronal signaling by affecting the expression of MEK, ERK, RSK and MSK, or lead to enhanced excitability of neurons, which plays an important role in synchronized discharge ${ }^{[3,9]}$. For example, Liu et al. ${ }^{[10]}$ found that the expression level of CHM 1 was significantly higher in the spinal dorsal horn of rats with painful diabetic neuropathy and negatively correlated with the $50 \%$ reduction threshold. In this study, the expression of CHM 1 in the trigeminal nucleus of the ION-CCI group was significantly increased on d 14, suggesting that CHM 1 may be involved in the development of pain behavior in $\mathrm{TN}$ rats.

Currently, no specific compound can regulate the expression level of CHM $1^{[11,12]}$. However, as CHM 1 acted as an ion channel to mediate ion influx, non- 
specific inorganic dyes such as $\mathrm{RR}$ and $\mathrm{Zn}^{2+}$ could block the action of CHM 1 by completely blocking the permeability of CHM $1^{[9]}$. In fact, in the model of cerebral hypoxic injury caused by clamping the middle cerebral artery, knocking out the CHM 1 gene or intraperitoneal injection of RR both can significantly reduce the infarct size ${ }^{[13]}$, suggesting that normal function of CHM 1 channel is a prerequisite for CHM 1 function. Thus, in this investigation RR was administered to investigate the role of CHM 1 in TN. The results indicated that after injection of RR, the threshold of mechanical pain was increased, while the number of scratches was decreased, which indicated that RR could alleviate hyperalgesia in $\mathrm{TN}$ rats. However, injection of RR had no effect on the expression of CHM 1. In conclusion, these results demonstrated that CHM 1 played an important role in altering the pain behavior in $\mathrm{TN}$ rats. Besides, injection of RR can improve hyperalgesia in TN rats without affecting the CHM 1 expression, which is expected to be a potential target for the treatment of TN.

\section{Conflict of interest:}

No conflict of interest between any of the authors.

\section{Acknowledgement}

This work was supported by the Human Provincial Natural Science Foundation of China (Grant No. 2019JJ40462)

\section{REFERENCES}

1. Ghurye S, McMillan R. Orofacial pain - an update on diagnosis and management. Br Dent J 2017;223:639-47.

2. Vingtdeux V, Chang EH, Frattini SA, Zhao H, Chandakkar P, Adrien L, et al. CHM 1 deficiency impairs cerebral neuron activity and memory flexibility in mice. Sci Rep 2016;6:1-4.

3. Ma Z, Siebert AP, Cheung KH, Lee RJ, Johnson B, Cohen AS, et al. Calcium homeostasis modulator 1 (CHM 1) is the poreforming subunit of an ion channel that mediates extracellular $\mathrm{Ca} 2+$ regulation of neuronal excitability. Proc Natl Acad Sci 2012;109:E1963-71.
4. Kernisant M, Gear RW, Jasmin L, Vit JP, Ohara PT. Chronic constriction Injury of the Infraorbital Nerve in the Rat using modified syringe needle. Neurosci Methods 2008;172:43-7.

5. Vos BP, Hans G, Adriaensen H. Behavioral assessment of facial pain in rats: face grooming patterns after painful and non-painful sensory disturbances in the territory of the rat's infraorbital nerve. Pain 199876:173-8.

6. Spradley JM, Davoodi A, Carstens MI. Opioid modulation of facial itches-and pain-related responses and grooming behavior in rats. Acta Derm Venereol 2012;92:515-20.

7. Fromm GH, Terrence CF, Maroon JC. Trigeminal neuralgia curren concepts regarding etiology and pathogenesis. Arch Neurol 1984;41:1204-7.

8. Kajander $\mathrm{KC}$, Bennett GJ. Onset of a painful peripheral neuropathy in rat: a partial and differential deafferentation and spontaneous discharge in A beta and A delta primary afferent neurons. J Neurophysiol 1992;68:734-44.

9. Dreses-Werringloer U, Vingtdeux V, Zhao H, Chandakkar P, Davies P, Marambaud P. CHM 1 controls the $\mathrm{Ca}^{2}$-dependent MEK, ERK, RSK and MSK signaling cascade in neurons. J Cell Sci 2013;126:1199-06.

10. Liu W, Ao Q, Guo Q, He W, Peng L, Jiang J, et al. miR-9 Mediates CHM 1-activated ATP-P2X7R signal in painful diabetic neuropathy rats. Mol Neurobiol 2017;54:922-9.

11. Vingtdeux V, Chandakkar P, Zhao H, Blanc L, Ruiz S, Marambaud P. CHM 1 ion channel elicits amyloid- $\beta$ clearance by insulin degrading enzyme in cell lines and in vivo in the mouse brain. J Cell Sci 2015;128:2330-38.

12. Moreno-Ortega AJ, Martínez-Sanz FJ, Lajarín-Cuesta R, de los Rios C, Cano-Abad MF. CGP37157 and its 20-isopropyl analogue modulate $\mathrm{Ca}^{2+}$ entry through $\mathrm{CHM} 1$. Neuropharmacol 2015;95:503-10.

13. Cisneros-Mejorado A, Gottlieb M, Ruiz A, Chara JC, PérezSamartín A, Marambaud P, et al. Blockade and knock-out of CHM 1 channels attenuate ischemic brain damage. J Cereb Blood Flow Metab 2018;38:1060-69.

This is an open access article distributed under the terms of the Creative Commons Attribution-NonCommercial-ShareAlike 3.0 License, which allows others to remix, tweak, and build upon the work non-commercially, as long as the author is credited and the new creations are licensed under the identical terms

This article was originally published in a special issue, "Recent Trends in Biomedical Research"

Indian J Pharm Sci 2020:82(1)spl issue1; XX-XX 